Jeroen J. G. Geurts

Erwin L. A. Blezer

Hugo Vrenken

Annette van der Toorn

Jonas A. Castelijns

Chris H. Polman

Petra J. W. Pouwels

Lars Bö

Frederik Barkhof

\section{Does high-field MR imaging improve cortical lesion detection in multiple sclerosis?}

Received: 15 December 2006

Received in revised form: 2 March 2007

Accepted: 5 March 2007

Published online: 4 February 2008

J. J. G. Geurts, PhD (区) • J. A. Castelijns •

F. Barkhof

VU University Medical Center

Dept. of Radiology, room PK 0X-112

De Boelelaan 1117

1081 HV Amsterdam, The Netherlands

Tel.: +31-20/444-0341

Fax: +31-20/444-2831

E-Mail: j.geurts@vumc.nl

H. Vrenken • P. J. W. Pouwels

VU University Medical Center

Dept. of Physics and Medical Technology

$1081 \mathrm{HV}$, Amsterdam, The Netherlands

C. H. Polman

VU University Medical Center

Dept. of Neurology

$1081 \mathrm{HV}$, Amsterdam, The Netherlands

J. J. G. Geurts · L. Bö

VU University Medical Center

Dept. of Pathology

$1081 \mathrm{HV}$, Amsterdam, The Netherlands

E. L. A. Blezer - A. van der Toorn

University Medical Center Utrecht

Image Sciences Institute

De Boelelaan 1117

Heidelberglaan 100

3584 CX, Utrecht, The Netherlands
Abstract Objective Cortical lesions in multiple sclerosis (MS) are notoriously difficult to visualize with standard MR imaging (MRI) techniques. However, the use of higher field-strengths with intrinsically higher signal-to-noise, which can partly be used to increase spatial resolution, may improve cortical lesion detection. Therefore, in this post mortem study, the sensitivity of high fieldstrength MRI (4.7 T) for cortical lesions was investigated, and compared to that of standard fieldstrength (1.5 T). Methods At 1.5 T, dual-echo T2-weighted spin-echo, as well as 3D-FLAIR images of seventeen formalin-fixed coronal MS and four control hemispheres were acquired. At $4.7 \mathrm{~T}$, the same specimens were imaged with a mainly proton-density (PD)weighted sequence. Proteolipid protein (PLP)-stained tissue sections $(10 \mu \mathrm{m})$ of the same brain slices were matched to the corresponding MR images, and cortical lesions were scored on all three MR sequences (blinded to histology) and in tissue sections (blinded to MRI). Sensitivity of the sequences for four cortical lesion types was calculated. Additionally, an unblinded, retrospective MR scoring was performed. Results Sensitivity for purely intracortical lesions (histological lesion types II, III, and IV; $\mathrm{n}=128$ ) was below $10 \%$ for both $1.5 \mathrm{~T}$ and $4.7 \mathrm{~T}$ MRI, while mixed gray matter-white matter (type I) lesions $(n=5)$ were detected in four out of five cases. All lesion counts increased upon retrospective (unblinded) scoring. However, up to $80 \%$ of the intracortical lesions still remained undetected. Conclusions MRI sensitivity for post mortem detection of cortical lesions is low, even when a higher field-strength was used. It varies, however, for different subtypes of cortical lesions.

Key words high-field MRI . sensitivity · cortical lesions . histopathology · type I lesions

\section{Introduction}

Multiple sclerosis (MS) is classically regarded as a disorder that predominantly affects the white matter (WM). However, involvement of the gray matter (GM) was already acknowledged in early pathology studies $[12,16]$ and has been reported with increasing fre- quency over the recent years. Histopathological detection of intracortical MS lesions has been improved by the use of myelin protein immunohistochemistry $[8,9$, $21,31]$, revealing a high prevalence of cortical MS lesions $[8,21,24,25]$.

Based on Dawson's initial observations [16], a classification system for cortical lesions has been proposed [8, 31], which was used in the current study. This system 
distinguishes between mixed GM-WM lesions (type I lesions) and purely intracortical lesions (types II-III-IV). Type II lesions are small intracortical lesions, usually surrounding a blood vessel; type III lesions are larger, extending from the pia downwards without reaching the subcortical WM; type IV lesions affect the entire width of the cortex from the pial surface down to the subcortical WM. Studies using standard histochemistry to describe cortical demyelination $[12,24]$ initially reported that type I lesions were most frequently observed, but more modern immunohistochemical methods revealed that type III lesions actually constitute the most common cortical lesion type [8, 21,31].

In a clinical setting, (juxta)cortical lesions are of diagnostic importance $[2,28]$, and WM abnormalities alone cannot explain the full range of symptoms observed in MS [1]. It has been hypothesized that pathology involving the GM may substantially contribute to clinical decline, and indeed juxtacortical lesions detected by magnetic resonance imaging (MRI) have been correlated to physical disability, cognitive impairment, and MS-associated epilepsy and depression [19, 26, 29, $32,34]$. Furthermore, changes in cortical volume [3, 14, 18] have been reported, as well as changes in quantitative MR measures of the "normal-appearing" cortical GM $[10,13,20,37]$, even in early phases of the disease [15]. To what extent those diffuse abnormalities can be explained by the presence of cortical lesions is as yet unclear, and in this perspective, it is essential to study the effects of cortical lesions (and their specific intracerebral distribution) on clinical deterioration, including neurocognitive deficits.

Unfortunately, however, studies that have correlated conventional MRI techniques to histopathology have proved that it is difficult to visualize lesions located within the cortical GM [21,24]. One of these studies [21] showed that up to $95 \%$ of the intracortical lesions as defined by histopathology, go undetected on MRI at $1.5 \mathrm{~T}$. Higher field-strengths with intrinsically higher signalto-noise ratio (SNR) may be expected to improve intracortical lesion detection.

Therefore, in the present study, high field-strength (4.7 T) MR images of 17 formalin-fixed coronal MS brain slices were compared to images obtained at standard field-strength $(1.5 \mathrm{~T})$, and the relative sensitivities of the different MR techniques for cortical lesions (types I-IV) were determined.

\section{Patients and methods}

\section{Patients and autopsy procedure}

Seventeen coronally cut $10-\mathrm{mm}$ thick hemispheric brain slices of ten chronic MS cases (seven females; mean age: 66.8 years) were selected after rapid autopsy (mean post mortem delay: $8 \mathrm{~h}$ and $25 \mathrm{~min}$ ), as well as four slices from three non-neurological controls (one female; mean age: 41.7 years). The slices were placed in a $10 \%$ formalin solution for several weeks, to allow for appropriate fixation. For details concerning demographic and neuropathologic data of patient and control

Table 1 Demographic and neuropathologic data

\begin{tabular}{llllllll}
\hline Case & No & Sex & Age & PMD & DD & Type & COD \\
\hline 1 & 2 & F & 74 & $06: 00$ & 15 & SP & cardiac arrest \\
2 & 1 & F & 48 & $04: 50$ & 20 & SP & euthanasia \\
3 & 2 & F & 72 & $12: 00$ & 14 & PP & pneumonia \\
4 & 1 & M & 77 & $04: 15$ & 27 & PP & stroke \\
5 & 1 & F & 48 & $05: 50$ & 18 & SP & cong. heart failure \\
6 & 2 & F & 65 & $06: 00$ & 25 & PR & cardiac failure \\
7 & 2 & F & 84 & $08: 45$ & 12 & SP & euthanasia \\
8 & 2 & M & 73 & $06: 45$ & 16 & SP & septicaemic shock \\
9 & 2 & M & 59 & $22: 15$ & 22 & SP & myocardial infarct \\
10 & 2 & F & 68 & $07: 30$ & 23 & SP & pneumonia \\
\hline Mean & & & 66.8 & $08: 25$ & 19.2 & & \\
(SD) & & & $(12.0)$ & $(05: 20)$ & $(5.0)$ & & cardiac failure \\
\hline 11 & 1 & $\mathrm{~F}$ & 57 & unknown & NA & control & Co + AC \\
12 & 2 & M & 22 & unknown & NA & control & HK \\
13 & 1 & M & 46 & unknown & NA & control & DC + PPH \\
\hline Mean & & & 41.7 & & & & \\
(SD) & & & $(17.9)$ & & & & \\
\hline
\end{tabular}

No number of hemispheric slices included (1 or 2) per case; $P M D$ post mortem delay (hrs:min); $D D$ disease duration (yrs); type type of disease (SP secondary progressive; PP primary progressive; $P R$ progressive relapsing; control non-neurological control); $C O D$ cause of death; $H K+A C$ hyperglycemic ketoacidosis (diabetes mellitus type I) in combination with alcoholic cardiomyopathy; $D C+P P H$ decompensatio cordis due to primary pulmonary hypertension; NA not applicable 
cases, see Table 1. Permission for performing autopsies, use of tissue and access to medical records for research purposes was granted by the local institutional ethics review board, and all subjects gave informed consent prior to death.

\section{Standard-field (1.5 T) and high-field (4.7 T) MR imaging}

The formalin-fixed brain slices were separately put into a purposebuilt perspex single-slice holder $\left(100 \times 70 \times 10 \mathrm{~mm}^{3}\right)$ and immersed in a non-magnetic oil (Fomblin; perfluorinated polyether, Solvay Solexis, Weesp, The Netherlands), after having removed the meninges. This way, inconvenient partial volume effects, resulting from suboptimal intrasulcal suppression of remaining small quantities of fixative and water, could be reduced. Furthermore, Fomblin has a low dielectric effect, which eliminates unwanted susceptibility artifacts near the boundaries of tissue samples. For measurements at clinical field-strength (1.5 T; Siemens Vision, Erlangen, Germany), the perspex single-slice holder was placed in a larger multi-slice holder, which fits exactly into the head coil of the MR system, as described before [6]. At 1.5 T, the sequences used were optimized for clinical scanning protocols. Dual-echo T2-weighted conventional spin-echo (T2SE) images (TR/TE/NEX: $2755 \mathrm{~ms} / 45$ and $90 \mathrm{~ms} / 2$; field of view $($ FoV): $80 \times 128 \mathrm{~mm}$; matrix size: $160 \times 256$; slice thickness: $3 \mathrm{~mm}$; acquisition time: $7 \mathrm{~min} 25 \mathrm{~s}$ ), as well as 3D fluid-attenuated inversion recovery (3D-FLAIR) images (TR/TE/TI/NEX: $6500 \mathrm{~ms} / 120 \mathrm{~ms} / 2200$ $\mathrm{ms} / 1$; 8 partitions per slab; partition thickness: $1.25 \mathrm{~mm}$; FoV: $125 \times 200 \mathrm{~mm}$; matrix size: $160 \times 256$; acquisition time: 7 min $54 \mathrm{~s}$ ) were obtained from each brain slice.

For measurements at high-field (experimental animal scanner, 4.7 T horizontal bore, Varian, Palo Alto, CA, USA), the holder was placed and fixed into a birdcage volume coil $(\varnothing 85 \mathrm{~mm}$, length $120 \mathrm{~mm}$ ). At $4.7 \mathrm{~T}$, the slices were imaged with a mainly proton density (PD)-weighted sequence (FSE3D; TR/TE/NEX: 4000 ms/9 ms/2; echo train length: 8; partition thickness: $0.39 \mathrm{~mm}$; 64 partitions per slab; FoV: $100 \times 100 \mathrm{~mm}$; matrix size: $256 \times 256$; acquisition time: $4 \mathrm{~h} 55$ min), which mimicked the appearance of the short echo of the T2weighted spin-echo sequence at $1.5 \mathrm{~T}$, with the combined benefit of better spatial resolution and higher SNR.
MR images of the controls were used to study normal appearance, as well as possible artifacts (Fig. 1A). Moreover, contrast-to-noise ratios (CNR) were determined for the different MR image types, on the basis of signal intensity (SI) measurements in regions-of-interest (i.e., GM, GM lesions, WM, WM lesions, Fomblin [noise]). The CNR between two tissue types was defined as: $\left|\mathrm{SI}_{1}-\mathrm{SI}_{2}\right| /$ standard deviation (noise), taking into account a correction for underestimation of the noise in magnitude images [23]. In this definition, the absolute difference of signal intensities is used, so that negative values in the numerator are accounted for. CNRs were calculated for WM and GM contrast (WM-GM CNR; both in MS and control samples), GM and GM lesions (GM-GML CNR; in MS samples only), and WM and WM lesions (WM-WML CNR; MS samples). For calculation of T2SE CNRs, the short-echo images were used.

\section{Histology and immunohistochemistry}

After the MR images had been obtained, the 10-mm thick formalinfixed brain slices were cut in half to reveal the imaged plane, and the $5-\mathrm{mm}$ slices were subsequently paraffin-embedded. Serial $10-\mu \mathrm{m}$ thick sections were cut, mounted onto Superfrost glass slides (Menzel-gläser), and dried overnight at $37^{\circ} \mathrm{C}$. Sections were deparaffinated in a series of xylene, $100 \%$ alcohol (ethanol), $96 \%$ alcohol, $70 \%$ alcohol and water. Standard Luxol Fast-Blue (LFB)-Periodic Acid Schiff (PAS) stainings were performed to enable visual matching of histological sections to the standard- and high-field MR images.

For immunohistochemistry, endogenous peroxidase activity was blocked by incubating the sections in methanol with $0.3 \% \mathrm{H}_{2} \mathrm{O}_{2}$. After having allowed the sections to cool sufficiently, they were rinsed. All washes were carried out for $30 \mathrm{~min}$ with $0.01 \mathrm{M}$ phosphatebuffered saline (PBS; pH 7.4). Antibodies were diluted in PBS containing $0.1 \%$ bovine serum albumin (BSA). To prevent non-specific binding, sections were preincubated with PBS containing $5 \%$ BSA for $10 \mathrm{~min}$ at room temperature.

Primary antibodies (Proteolipid Protein (PLP), Serotec, Oxford, UK) were diluted 1:3000 in PBS-BSA and incubated for $1 \mathrm{~h}$ at room temperature. After washing, immunolabelling with primary antibodies was detected with biotinylated rabbit anti-mouse (1:500) for
Fig. 1 Examples of a control and an MS brain slice at 4.7 T PD; A coronal 4.7T PD image of a control brain slice; arrowheads point out widened Virchow-Robin spaces, which could be distinguished morphologically from intracortical MS lesions; B coronal 4.7 T PD image of an MS brain slice, showing both focal WM lesions (asterisks) and diffuse (perilesional) WM abnormalities (arrowhead) in detail. The arrows point out areas that are more difficult to interpret due to field inhomogeneities, decreasing the signal in the upper and lower extremities of the slice
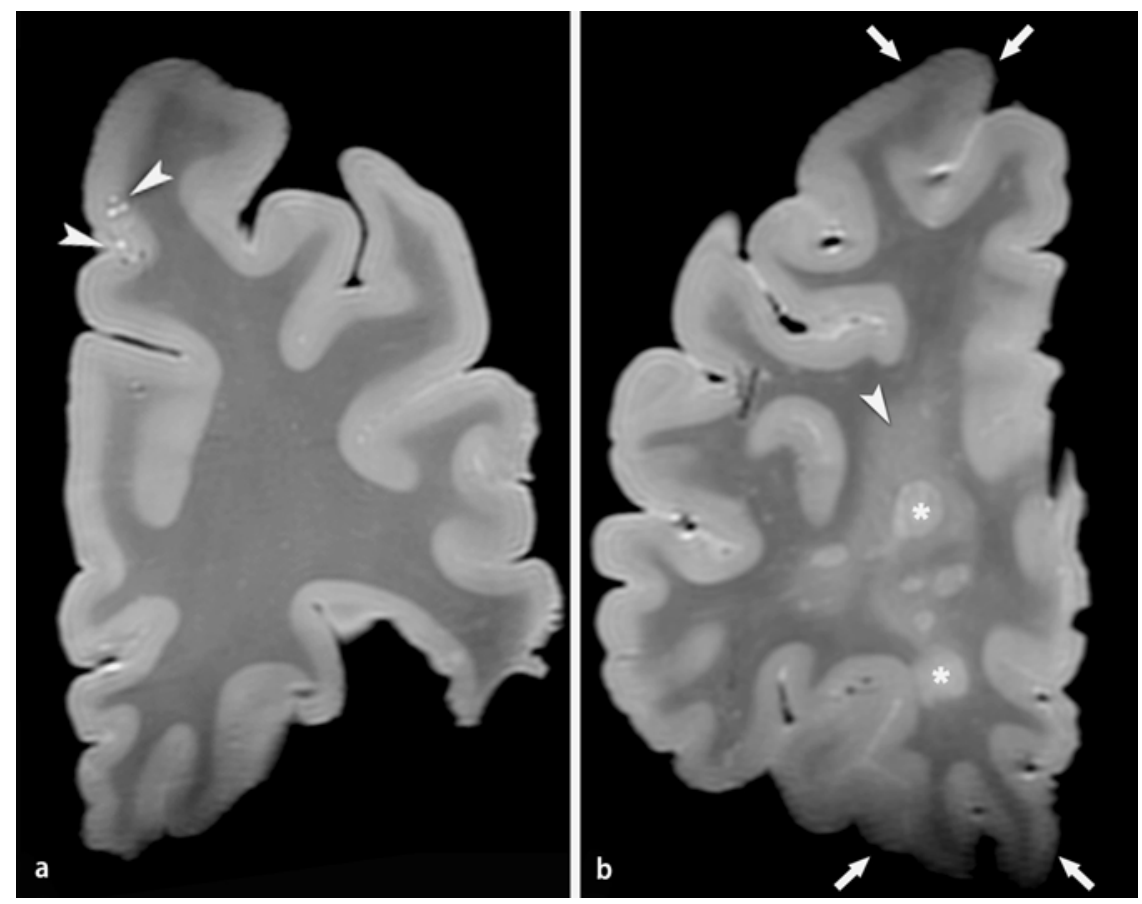
$30 \mathrm{~min}$ at room temperature and avidin-biotin-peroxidase complexes (sABC-HRP 1:200, DAKO, Glostrup, Denmark) for $60 \mathrm{~min}$ at room temperature. Peroxidase activity was demonstrated with $0.5 \mathrm{mg} / \mathrm{ml}$ 3,3'diaminobenzidinetetrahydrochloride (DAB; Sigma, St. Louis, MO) in PBS containing $0.03 \% \mathrm{H}_{2} \mathrm{O}_{2}$ for $5 \mathrm{~min}$, which leads to a brown reaction product. Sections were lightly counterstained with haematoxylin and mounted with Depex (BDH, Poole, UK).

\section{MRI to histopathology matching and analysis}

Hemispheric tissue sections were carefully matched to the corresponding MR imaging planes of the $1.5 \mathrm{~T}$ and $4.7 \mathrm{~T}$ images, using cortical anatomy and WM lesions as landmarks $[6,21]$. An example of an LFB-PAS stained tissue section that was successfully matched to the corresponding 4.7 T PD image is shown in Fig. 2.

Numbers of cortical lesions were scored by the MR reader, first on the short-echo of the $1.5 \mathrm{~T}$ T2SE, then on the $1.5 \mathrm{~T}$ 3D-FLAIR, and finally on the $4.7 \mathrm{~T}$ PD images (allowing for appropriate time intervals between scorings to avoid recall bias). The following criteria were used to define cortical lesions: 1) lesions should appear hyperintense on MRI, intermediate to the signal intensities of WM lesions and adjacent normal cortex; 2) lesional borders should be irregularly shaped (as in histology), and 3) lesions should not be clearly traceable over several subsequent slices (to avoid aberrant scoring of vascular structures). The MR reader's scoring was blinded to histopathology. Not all cortical areas studied histologically could be analyzed on the MR images, since field inhomogeneities at the upper and lower boundaries of the (larger) brain slices (especially at 4.7T) occasionally caused the images to be poorly interpretable in these areas (illustrated in Fig. 1B). Regions with such signal loss in any MR image type were excluded from all analyses. The pathology reader also scored cortical lesion numbers (blinded to MR data) and classified them (mixed GMWM or type I lesions; intracortical lesions, types II, III or IV $[9,21$, 31]), using the PLP-stained tissue sections. Finally, total numbers of type I-IV lesions scored with the different MR sequences were expressed as percentages of the total number of lesions scored histologically (i.e. the sensitivity of the three MR sequences for the different cortical lesion types). After the blinded scoring of the MR images, precise localizations of all lesions were revealed to the MR reader and a second (retrospective) assessment of the images was performed.

\section{Results}

Normal appearance of the three MR sequences (1.5 T T2SE, 1.5 T 3D-FLAIR and 4.7 T PD) was investigated and artifacts were marked. In Fig. 1 A, cortical VirchowRobin spaces can be recognized as punctate hyperintensities on a $4.7 \mathrm{~T}$ PD image of a control brain slice. On 4.7 T MR images, these areas could be distinguished morphologically from focal cortical lesions in the MS brain slices. In total, 27 cortical lesions, of which 26 type III lesions and one type IV lesion had to be excluded from further analysis, due to local field inhomogeneities at $4.7 \mathrm{~T}$ and subsequent poor visibility of these lesions on scans.

In the MS brain slices, a total of 133 cortical lesions were detected, only five of which were mixed GM-WM (type I) lesions. The total number of lesions that were located entirely within the cortical GM (intracortical types II, III, and IV lesions) was therefore 128, most of which $(\mathrm{n}=101)$ consisted of type III (subpial) lesions, followed in number by the smaller type II lesions $(n=22)$, and the cortex-spanning type IV lesions $(n=5)$. Table 2 shows the numbers of lesions found in the tissue sections, as well as the proportions of those lesions detected by standard- and high-field MRI.

On both standard-field and high-field MR sequences, nearly all of the type I lesions were seen (one lesion was not scored prospectively on 3D-FLAIR; see Table 2 ). However, the intracortical part of mixed lesions was often most clear on the high-field PD sequence (Fig. 3). Intracortical lesions (types II, III, and IV) could be identified in a minority of cases only (Table 2, Fig.4). Prospectively, the sensitivity of the sequences for these lesions at both field-strengths remained below $10 \%$.
Fig. 2 Matching of a Luxol Fast-Blue (LFB) - Periodic Acid Schiff (PAS) stained hemispheric tissue section (right) to the 4.7 T PD image of the same brain slice (left). The visual matching procedure relies on the presence of different anatomical landmarks, and a high accuracy could be achieved (for example, see the anatomical linings of claustrum and pontes grisei of the lentiform nucleus). Areas that were damaged (black arrow) or otherwise difficult to match (white arrows) were excluded from analysis
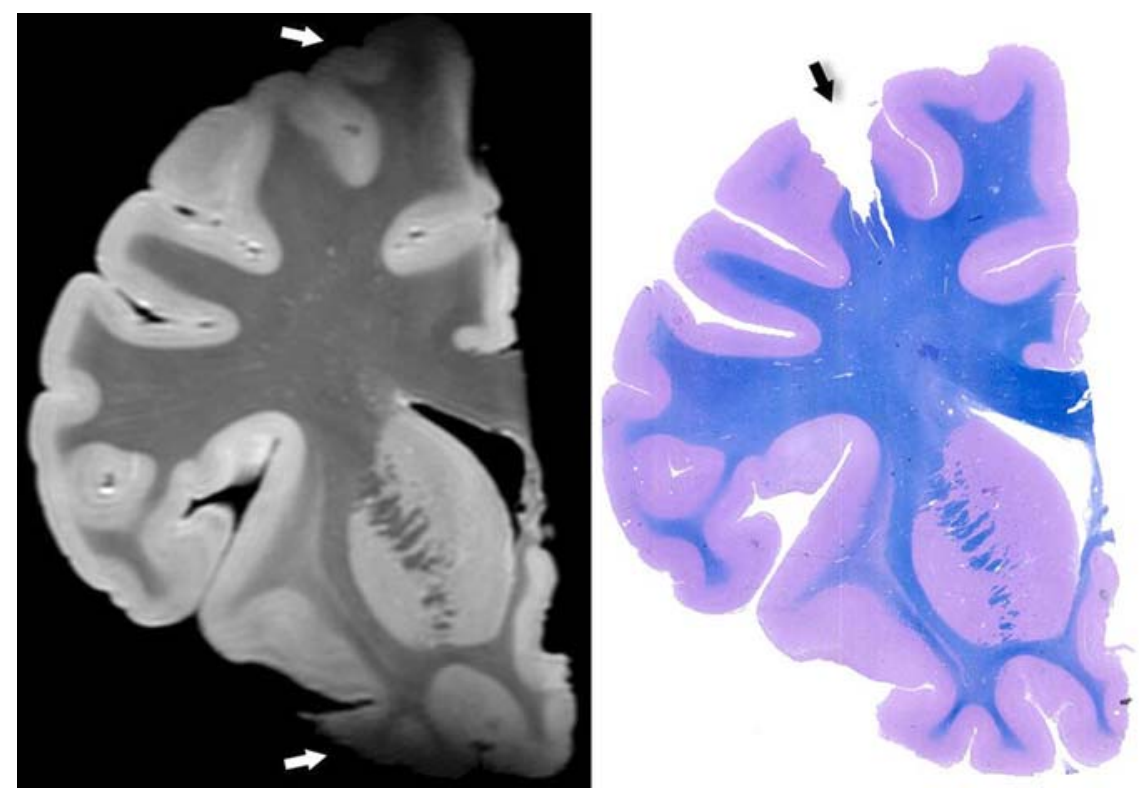
Table 2 Number of cortical lesions found with standard- (1.5T) and high-field (4.7T) MRI, and sensitivities of the different MR sequences for the different cortical lesion types

\begin{tabular}{|c|c|c|c|c|c|c|c|}
\hline \multirow{3}{*}{ lesion type } & \multirow{3}{*}{$\mathrm{N}$} & \multicolumn{3}{|c|}{ Prospective scoring } & \multicolumn{3}{|c|}{ Retrospective scoring } \\
\hline & & \multicolumn{2}{|l|}{ 1.5T MRI } & \multirow{2}{*}{$\frac{4.7 T \mathrm{MRI}}{\mathrm{PD}}$} & \multicolumn{2}{|l|}{$1.5 \mathrm{~T} \mathrm{MRI}$} & \multirow{2}{*}{$\frac{4.7 \mathrm{~T} M R I}{\mathrm{PD}}$} \\
\hline & & T2SE & 3D-FLAIR & & T2SE & 3D-FLAIR & \\
\hline Type I & 5 & $5(100)$ & $4(80)$ & $5(100)$ & $5(100)$ & $5(100)$ & $5(100)$ \\
\hline Type II & 22 & $1(4.5)$ & $2(9.1)$ & $3(13.6)$ & $7(31.8)$ & $6(27.3)$ & $7(31.8)$ \\
\hline Type III & 101 & $8(7.9)$ & $9(8.9)$ & $4(4.0)$ & $29(28.7)$ & 19 (18.8) & $17(16.8)$ \\
\hline Type IV & 5 & $2(40)$ & $1(20)$ & $0(0)$ & $4(80)$ & $2(40)$ & $4(80)$ \\
\hline Type II-III-IV & 128 & $11(8.6)$ & $12(9.4)$ & $7(5.5)$ & $40(31.3)$ & $27(21.1)$ & $28(21.9)$ \\
\hline
\end{tabular}

Prospective scoring blinded (to histology) MRI scoring of cortical lesion types; retrospective scoring unblinded MRI scoring; $N$ total number of lesions (per lesion type) found histopathologically; T2SE, 3D-FLAIR and PD numbers of lesions as detected by these sequences; the sensitivity (number of lesions scored with the respective MRI techniques as a percentage of the total number of lesions found in histology) is shown between brackets. Type II-IIIIV is the combined number of intracortical lesions
Table 3 Contrast-to-noise ratios (SD)

\begin{tabular}{lrrrl}
\hline \multirow{2}{*}{ Contrast-to-noise ratio (CNR) } & \multicolumn{1}{l}{$1.5 \mathrm{~T}$} & & \multicolumn{2}{l}{$4.7 \mathrm{~T}$} \\
\cline { 2 - 3 } \cline { 5 - 6 } \cline { 5 - 6 } & \multicolumn{1}{c}{ T2SE } & 3D-FLAIR & & PD \\
\hline WM-GM & $9.4(1.8)$ & $6.3(2.1)$ & & $64.2(13.1)$ \\
WM-WML & $13.6(4.4)$ & $21.4(8.4)$ & & $79.5(28.4)$ \\
GM-GML & $2.8(2.0)$ & $1.7(0.9)$ & & $2.3(2.7)$ \\
\hline
\end{tabular}

WM-GM white matter to gray matter CNR; WM-WML white matter to white matter lesion CNR; GM-GML gray matter to gray matter lesion CNR

Upon retrospective scoring, lesion counts increased as expected, but still up to about $80 \%$ of the intracortical lesions could not be detected, even though the exact localization of the lesions had at that time been revealed to the MR reader. The differences between MR sequences and field-strengths, in terms of sensitivity for the separate intracortical lesion types, were minute (Table 2). Therefore, data were presented purely descriptively.

The 4.7 T PD images showed highest WM-GM and WM-WML CNRs. However, GM-GML CNRs of all three sequences (both field-strengths) were comparably low (Table 3), indicating that the contrast between GM and GM lesions is very low, independent of sequence or fieldstrength (for an illustration, see Fig. 4 and also the intracortical part of the type I lesion shown in Fig. 3).

\section{Discussion}

This study shows that the sensitivity of MRI for cortical lesions is low, even when using higher field-strengths to improve spatial resolution and SNR. However, MR sensitivity depends on lesion type: mixed type I lesions can be visualized relatively well at both field-strengths, while intracortical lesions (histopathological types II, III, and IV) are seen in less than $10 \%$ of the cases.

The use of specific myelin immunohistochemistry (like PLP and Myelin Basic Protein [MBP]), as opposed to standard histochemistry (Luxol Fast-Blue [LFB]), has greatly contributed to the detection of (intra)cortical lesions in post mortem MS brain tissue. However, increased detection of GM lesions under the microscope has also made clear that the majority of these lesions are missed by (standard) in vivo MR imaging techniques $[21,24]$. This hinders further research into the clinical effects of GM abnormalities, as well as more fundamental studies focusing on pathogenetic mechanisms of GM pathology.

Low sensitivity of standard MRI for cortical lesions was already reported in an earlier study [21], and the standard-field results in the present study, obtained from a different material (different patients and formalin-fixed instead of fresh tissue), are largely in agreement with those previous results. In that same study, it was hypothesized that imaging at higher field-strengths may be beneficial when trying to image (intra)cortical lesions. In the current study, 4.7 T MR imaging was used to substantially improve spatial resolution, in combination with good SNR. However, as already mentioned and contrary to our expectations, detection of (intra)cortical lesions was not increased. Since the observed cortical lesion numbers were too small, no statistical analysis was performed, and the seemingly different numbers in Table 2 should be cautiously interpreted.

Several factors may underlie the low sensitivity of MRI for cortical lesions. For example, purely intracortical lesions were reported to be largely non-inflammatory $[8,11]$ and neuroaxonal elements seem to be relatively preserved $[5,16]$, although they may still be affected to some degree $[31,35,38]$. In addition, because of low intrinsic myelin, demyelination in the subpial layers will only have a very limited effect on the T2-relaxation times. As a result of these effects, intracortical lesions may generate only little contrast on MRI. This seems to be confirmed by the low GM-GML CNRs at both $1.5 \mathrm{~T}$ and $4.7 \mathrm{~T}$ MRI. It should be noted, however, that although CNRs have been calculated, the goal of this 
Fig.3 Example of a mixed GM-WM (type I) lesion in a coronally cut MS brain slice; A T2SE image of part of the top area of an MS brain slice showing a type I lesion (arrow). The GM part of the lesion is difficult to appreciate; B same area (arrow) on 3D-FLAIR: again the WM part is better visible than the GM part; $\mathbf{C}$ highfield PD MR image of the same lesion (arrow). The intracortical part of the lesion can now be slightly better distinguished (arrowheads); D PLP stain (2.5x magnification) of the same (matched) area, showing in detail the demyelination in both the cortical GM (arrowheads) and subcortical WM (arrow), as was already visible on the $4.7 \mathrm{TPD}$
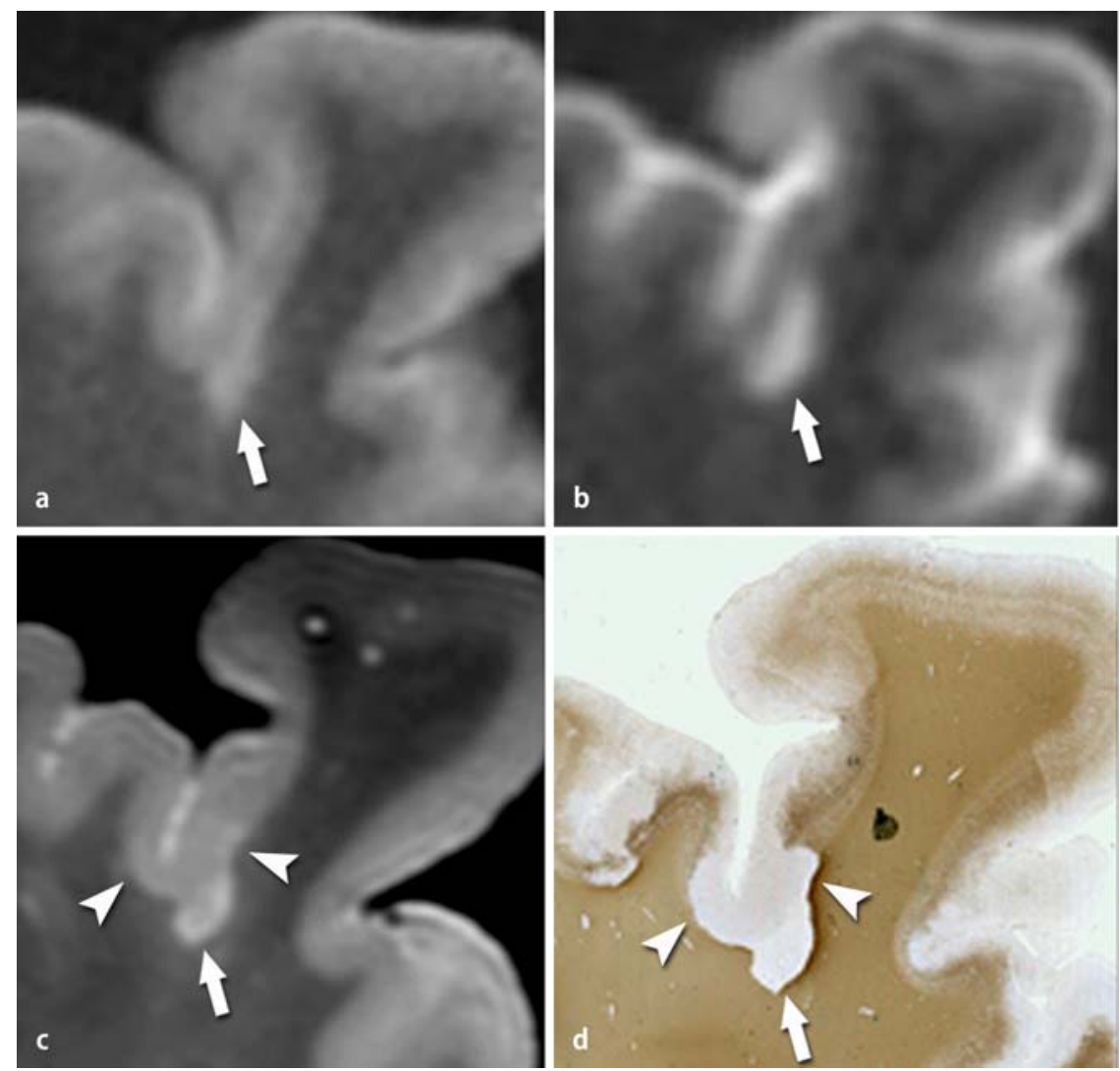

study was not to quantitatively compare CNRs between sequences or field strengths. Instead, we merely aimed to improve cortical lesion detection by using high-field MRI to predominantly increase spatial resolution, using standardly available imaging sequences. Clinical fieldstrength $(1.5 \mathrm{~T})$ was used as a reference, and sequence parameters at $4.7 \mathrm{~T}$ were chosen such that both spatial resolution and SNR were enhanced compared to $1.5 \mathrm{~T}$. Obviously, voxel dimensions and acquisition times were different, and contrast can be expected to decrease due to shortening of T2-relaxation times at higher fieldstrengths. T1- and T2-relaxation times are also subject to change following death and fixation $[4,27,30]$, and the clinical sequences used in this study were not specifically optimized for the post mortem situation. These methodological limitations are likely to have contributed to some extent to the observed insensitivity of the MR techniques to cortical lesions. This also holds for partial volume effects with fluid surrounding the brain slices, which may hamper adequate cortical lesion detection [21]. The non-magnetic oil Fomblin, in which the slices were completely immersed, was used in an attempt to eliminate the high signal intensity from the watery fluid in the sulci. In the narrower sulci, however, optimal results could not always be obtained (data not shown); this may create problems, especially when trying to vi- sualize the superficially localized (type III) lesions. The fact that type III lesions are particularly difficult to image is unfortunate, since it is the most abundant intracortical lesion type $[9,21,31]$, which may occasionally extend over several gyri, resulting in a pattern of general cortical subpial demyelination $[7,9,25]$.

Further difficulties in post mortem MRI-histopathology correlative studies may be expected in matching tissue sections (of $10 \mu \mathrm{m}$ thickness in this study) to post mortem MR images (with slice thickness in the mmrange in this study). However, with the large hemispheric samples used in the current study, highly accurate matching could be obtained, since many different anatomical landmarks could be used (see Fig. 2 and also [6]). Areas that could still not be matched (due to e.g. small deviations in angulation during the sectioning process) were excluded from analysis.

As opposed to the intracortical lesion types II, III and IV, type I lesions were easier to detect, both at standardand at high-field. Apart from the fact that the more conspicuous WM part of type I lesions generally draws the observer's eye to the spot, the GM part of type I lesions is also usually more easy to distinguish on MRI than the purely intracortical lesions. As mentioned before, this may be essentially related to the fact that the lower layers of the cortical GM are more densely myelinated than 
Fig.4 An example of an intracortical (type III) lesion in a coronally cut MS brain slice; A T2SE image showing no signal abnormalities in the cortex. 3D-FLAIR image (B) and 4.7T PD image (C) of the same area, also showing a lack of pathology; PLP stain (D) (2.5x magnification) of same (matched) area: whereas none of the MR images show any signal abnormalities in this region, an extensive type III lesion is histologically apparent (border between normal and demyelinated cortex indicated by the arrows). This figure clearly illustrates the lack of conspicuity of both standard- and high-field MRI for intracortical demyelination, as reported in the current study
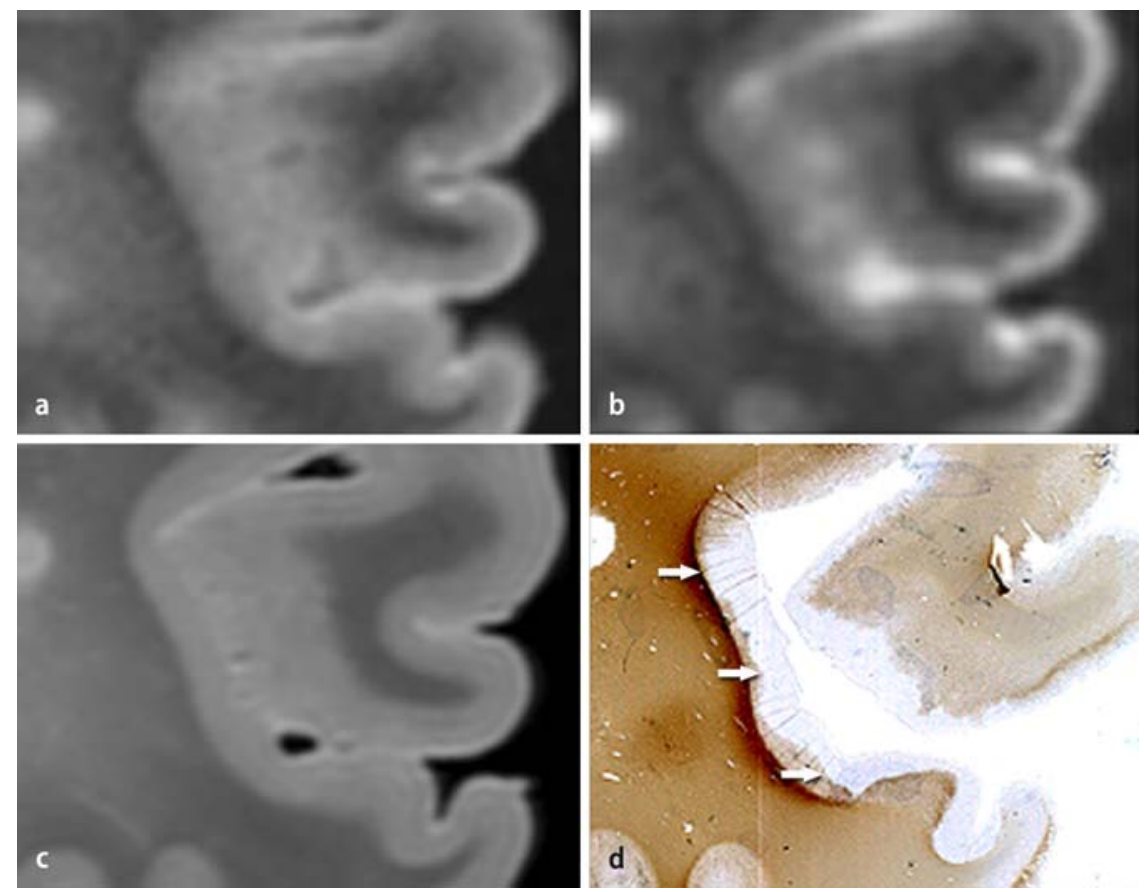

the upper layers, so that demyelination within these areas may create greater $\mathrm{T} 2$ contrast. It has also been shown that type I lesions, including the GM part, may exhibit higher numbers of T-lymphocytes and of activated microglia/macrophages than the purely intracortical lesion types $[8,11]$. Whether increased inflammation within type I cortical lesions is responsible for the higher MR conspicuity of these lesions could not be determined in the current study. This remains an important issue for future research.

It has already been shown on several occasions that focal MS lesions in the WM are easy to image with standard MR methods in the post mortem setting $[17,21,24$, $33,36]$. Therefore, WM lesion detection was not specifically addressed in this study. However, both focal and diffuse WM abnormalities could be visualized well with all sequences (for an example of WM hyperintensities as visualized by 4.7T PD, see Fig. 4B), and WM-WML CNRs of all three sequences were high.

In the search for imaging modalities that allow for improved intracortical lesion detection, newly developed techniques such as Double Inversion Recovery (DIR), suppressing both WM and cerebrospinal fluid, may prove to be beneficial. In vivo, a multi-slab 3D-DIR sequence was recently reported to increase the detection of intracortical lesions and improve the distinction between the purely intracortical lesions and mixed GMWM lesions [22]. Adapting this particular sequence for post mortem purposes may be helpful. Imaging brain slices at even higher field-strengths than those used in the current study should also be considered. For the radiological practice, results thus obtained might be interesting in the long run, when stronger magnets (e.g. $7 \mathrm{~T}$ ) will have become more widely available.

Acknowledgments The authors would like to thank Mrs. Christa van Urk - van den Berg for excellent technical support and the Netherlands Brain Bank for supply of the brain material. J.J.G. Geurts (grant numbers: 00-427 and 02-358b) and L. Bö (grant number: $02-358 \mathrm{~b}$ ) were sponsored by the Dutch MS Research Foundation, Voorschoten, The Netherlands. 


\section{References}

1. Barkhof F (2002) The clinico-radiological paradox in multiple sclerosis revisited. Curr Opin Neurol 15:239-245

2. Barkhof F, Filippi M, Miller DH, Scheltens P, Campi A, Polman CH, Comi G, Ader HJ, Losseff N, Valk J (1997) Comparison of MRI criteria at first presentation to predict conversion to clinically definite multiple sclerosis. Brain 120:2059-2069

3. Benedict RH, Zivadinov R, Carone DA, Weinstock-Guttman B, Gaines J, Maggiore C, Sharma J, Tomassi MA, Bakshi $\mathrm{R}$ (2005) Regional lobar atrophy predicts memory impairment in multiple sclerosis. AJNR Am J Neuroradiol 26: 1824-1831

4. Blamire AM, Rowe JG, Styles P, McDonald B (1999) Optimising imaging parameters for post mortem MR imaging of the human brain. Acta Radiol 40:593-597

5. Bo L, Geurts JJ, Mork SJ, van der Valk P (2006) Grey matter pathology in multiple sclerosis. Acta Neurol Scand Suppl 183:48-50

6. Bo L, Geurts JJ, Ravid R, Barkhof F (2004) Magnetic resonance imaging as a tool to examine the neuropathology of multiple sclerosis. Neuropathol Appl Neurobiol 30:106-117

7. Bo L, Geurts JJ, van der Valk P, Polman C, Barkhof F (2007) Lack of correlation between cortical demyelination and white matter pathologic changes in multiple sclerosis. Arch Neurol 64: $76-80$

8. Bo L, Vedeler CA, Nyland H, Trapp BD, Mork SJ (2003) Intracortical multiple sclerosis lesions are not associated with increased lymphocyte infiltration. Mult Scler 9:323-331

9. Bo L, Vedeler CA, Nyland HI, Trapp BD, Mork SJ (2003) Subpial demyelination in the cerebral cortex of multiple sclerosis patients. J Neuropathol Exp Neurol 62:723-732

10. Bozzali M, Cercignani M, Sormani MP, Comi G, Filippi M (2002) Quantification of brain gray matter damage in different MS phenotypes by use of diffusion tensor MR imaging. AJNR Am J Neuroradiol 23:985-988

11. Brink BP, Veerhuis R, Breij EC, van der Valk P, Dijkstra CD, Bo L (2005) The pathology of multiple sclerosis is location-dependent: no significant complement activation is detected in purely cortical lesions. J Neuropathol Exp Neurol 64:147-155

12. Brownell B and Hughes JT (1962) The distribution of plaques in the cerebrum in multiple sclerosis. J Neurol Neurosurg Psychiatry 25:315-320
13. Cercignani M, Bozzali M, Iannucci G, Comi G, Filippi M (2001) Magnetisation transfer ratio and mean diffusivity of normal appearing white and grey matter from patients with multiple sclerosis. J Neurol Neurosurg Psychiatry 70:311-317

14. Dalton CM, Chard DT, Davies GR, Miszkiel KA, Altmann DR, Fernando K, Plant GT, Thompson AJ, Miller DH (2004) Early development of multiple sclerosis is associated with progressive grey matter atrophy in patients presenting with clinically isolated syndromes. Brain 127:1101-1107

15. Davies GR, Ramio-Torrenta L, Hadjiprocopis A, Chard DT, Griffin CM, Rashid W, Barker GJ, Kapoor R, Thompson AJ, Miller DH (2004) Evidence for grey matter MTR abnormality in minimally disabled patients with early relapsing-remitting multiple sclerosis. J Neurol Neurosurg Psychiatry 75:998-1002

16. Dawson JW (1916) The Histology of Multiple Sclerosis. Trans R Soc Edinburgh 50:517-740

17. De Groot CJ, Bergers E, Kamphorst W, Ravid R, Polman CH, Barkhof F, van der Valk P (2001) Post-mortem MRIguided sampling of multiple sclerosis brain lesions: increased yield of active demyelinating and $(\mathrm{p})$ reactive lesions. Brain 124:1635-1645

18. De Stefano N, Matthews PM, Filippi M, Agosta F, De Luca M, Bartolozzi ML, Guidi L, Ghezzi A, Montanari E, Cifelli A, Federico A, Smith SM (2003) Evidence of early cortical atrophy in MS: relevance to white matter changes and disability. Neurology 60:1157-1162

19. Feinstein A, Roy P, Lobaugh N, Feinstein K, O'Connor P, Black S (2004) Structural brain abnormalities in multiple sclerosis patients with major depression. Neurology 62:586-590

20. Ge Y, Grossman RI, Udupa JK, Babb JS, Kolson DL, McGowan JC (2001) Magnetization transfer ratio histogram analysis of gray matter in relapsingremitting multiple sclerosis. AJNR Am J Neuroradiol 22:470-475

21. Geurts JJ, Bo L, Pouwels PJ, Castelijns JA, Polman CH, Barkhof F (2005) Cortical lesions in multiple sclerosis: combined postmortem MR imaging and histopathology. AJNR Am J Neuroradiol 26:572-577

22. Geurts JJ, Pouwels PJ, Uitdehaag BM, Polman CH, Barkhof F, Castelijns JA (2005) Intracortical lesions in multiple sclerosis: improved detection with 3D double inversion-recovery MR imaging. Radiology 236:254-260
23. Henkelman RM (1985) Measurement of signal intensities in the presence of noise in MR images. Med Phys 12: 232-233

24. Kidd D, Barkhof F, McConnell R, Algra PR, Allen IV, Revesz T (1999) Cortical lesions in multiple sclerosis. Brain 122:17-26

25. Kutzelnigg A, Lucchinetti CF, Stadelmann C, Bruck W, Rauschka H, Bergmann M, Schmidbauer M, Parisi JE, Lassmann H (2005) Cortical demyelination and diffuse white matter injury in multiple sclerosis. Brain 128:2705-2712

26. Lazeron RH, Langdon DW, Filippi M, van Waesberghe JH, Stevenson VL, Boringa JB, Origgi D, Thompson AJ, Falautano M, Polman CH, Barkhof F (2000) Neuropsychological impairment in multiple sclerosis patients: the role of (juxta)cortical lesion on FLAIR. Mult Scler 6:280-285

27. Macchi G and Cioffi RP (1992) An in vivo and post mortem MRI study in multiple sclerosis with pathological correlation. Ital J Neurol Sci 13:97-103

28. McDonald WI, Compston A, Edan G, Goodkin D, Hartung HP, Lublin FD, McFarland HF, Paty DW, Polman CH, Reingold SC, Sandberg-Wollheim M, Sibley W, Thompson A, van den Noort S, Weinshenker BY, Wolinsky JS (2001) Recommended diagnostic criteria for multiple sclerosis: guidelines from the International Panel on the diagnosis of multiple sclerosis. Ann Neurol 50: 121-127

29. Moriarty DM, Blackshaw AJ, Talbot PR, Griffiths HL, Snowden JS, Hillier VF, Capener S, Laitt RD, Jackson A (1999) Memory dysfunction in multiple sclerosis corresponds to juxtacortical lesion load on fast fluid-attenuated inversion-recovery MR images. AJNR Am J Neuroradiol 20:1956-1962

30. Nagara $H$, Inoue $T$, Koga $T$, Kitaguchi T, Tateishi J, Goto I (1987) Formalin fixed brains are useful for magnetic resonance imaging (MRI) study. J Neurol Sci 81:67-77

31. Peterson JW, Bo L, Mork S, Chang A, Trapp BD (2001) Transected neurites, apoptotic neurons, and reduced inflammation in cortical multiple sclerosis lesions. Ann Neurol 50:389-400

32. Rovaris M, Filippi M, Minicucci L, Iannucci G, Santuccio G, Possa F, Comi G (2000) Cortical/subcortical disease burden and cognitive impairment in patients with multiple sclerosis. AJNR Am J Neuroradiol 21:402-408 
33. Schmierer K, Scaravilli F, Altmann DR, Barker GJ, Miller DH (2004) Magnetization transfer ratio and myelin in postmortem multiple sclerosis brain. Ann Neurol 56:407-415

34. Sokic DV, Stojsavljevic N, Drulovic J, Dujmovic I, Mesaros S, Ercegovac M, Peric V, Dragutinovic G, Levic Z (2001) Seizures in multiple sclerosis. Epilepsia 42:72-79
35. Vercellino M, Plano F, Votta B, Mutani R, Giordana MT, Cavalla P (2005) Grey matter pathology in multiple sclerosis. J Neuropathol Exp Neurol 64: 1101-1107

36. Vos CM, Geurts JJ, Montagne L, van Haastert ES, Bo L, van der Valk P, Barkhof F, de Vries HE (2005) Blood-brain barrier alterations in both focal and diffuse abnormalities on postmortem MRI in multiple sclerosis. Neurobiol Dis 20:953-960
37. Vrenken H, Pouwels PJ, Geurts JJ, Knol DL, Polman CH, Barkhof F, Castelijns JA (2006) Altered diffusion tensor in multiple sclerosis normal-appearing brain tissue: cortical diffusion changes seem related to clinical deterioration. J Magn Reson Imaging 23:628-636

38. Wegner C, Esiri MM, Chance SA, Palace J, Matthews PM (2006) Neocortical neuronal, synaptic, and glial loss in multiple sclerosis. Neurology 67: 960-967 